Research Article

\title{
The Maschke-Type Theorem and Morita Context for BiHom- Smash Products
}

\author{
Bingliang Shen ${ }^{1}$ and Ling Liu $\mathbb{D}^{2}$ \\ ${ }^{1}$ Shanghai University of Finance \& Economics Zhejiang College, Jinhua 321013, China \\ ${ }^{2}$ College of Mathematics and Computer Science, Zhejiang Normal University, Jinhua 321004, China
}

Correspondence should be addressed to Ling Liu; ntliulin@zjnu.cn

Received 3 November 2020; Revised 29 December 2020; Accepted 31 December 2020; Published 15 January 2021

Academic Editor: Yao Zhong Zhang

Copyright ( 2021 Bingliang Shen and Ling Liu. This is an open access article distributed under the Creative Commons Attribution License, which permits unrestricted use, distribution, and reproduction in any medium, provided the original work is properly cited.

Let $\left(H, \alpha_{H}, \beta_{H}, \omega_{H}, \psi_{H}, S_{H}\right)$ be a BiHom-Hopf algebra and $\left(A, \alpha_{A}, \beta_{A}\right)$ be an $\left(H, \alpha_{H}, \beta_{H}\right)$-module BiHom-algebra. Then, in this paper, we study some properties on the BiHom-smash product $A \# H$. We construct the Maschke-type theorem for the BiHomsmash product $A \# H$ and form an associated Morita context $\left[A^{H},{ }_{A^{H}} A_{A \# H}, A \# H, A_{A^{H}}, A \# H\right]$.

\section{Introduction}

The first instance of Hom-type algebras appeared in the physics literature when looking for quantum deformations of some algebras of vector fields in 1990's, such as Witt and Virasoro algebras $([1,2])$. This kind of algebras obtained by deforming certain Lie algebras no longer satisfied the Jacobi identity, but a modified version of it involving a homomorphism. Such algebra (called Hom-Lie algebra) was given in $[3,4]$. The associative counterpart of Hom-Lie algebras has been introduced in [5] (called Hom-associative algebras), and Hom-analogues of other algebraic structures have been introduced afterwards, Hom-coassociative coalgebras, Hombialgebras, Hom-pre-Lie algebras etc.

A categorical approach to Hom-type algebras was considered in [6]. A generalization has been given in [7], where a construction of a Hom-category including a group action led to concepts of BiHom-type algebras. Hence, BiHomassociative algebras and BiHom-Lie algebras, involving two linear maps (called structure maps), were introduced. The main tool to obtain examples of Hom-algebras from classical algebras, the so-called Yau twisting, works perfectly fine also in the BiHom-type case. There is a growing literature on Hom and BiHom-type algebras, and let us just mention the very recent papers [8-12].
Let $H$ be a Hopf algebra and $A$ an $H$-module algebra; then, as well known, we can construct the smash product algebra $A \# H$ (see [13] or [14]). Smash products plays an important role in the lifting method for the classification of finite-dimensional pointed Hopf algebras (see [15]). The Hom-forms of the smash product can be found in the following literature. In [16], the Maschke-type theorem for the Hom-smash product is given, and the Morita context is constructed. In [7], the authors defined the BiHom-smash product and gave some examples. Now, it is natural to ask how to prove the Maschke-type theorem and construct the associated Morita context for the BiHom-smash product?

The main aim of this paper is to give a positive answer to the above questions. We use the same strategy as in the Hom case and get an analogue of the Maschke-type theorem and form an associated Morita context between the BiHomsmash product and its BiHom-subalgebra in the setting of BiHom-Hopf algebras.

This paper is organized as follows. In Section 2, we recall some definitions and basic results related to BiHom-algebras, BiHom-coalgebras, BiHom-bialgebras, BiHom-modules, and module BiHom-algebras. In Sections 3 and 4, we study some properties on the BiHom-smash product $A \# H$. If $\left(H, \mu_{H}\right.$, $\left.\Delta_{H}, \alpha_{H}, \beta_{H}, \psi_{H}, \omega_{H}\right)$ is a finite-dimensional semisimple BiHom-Hopf algebra, then we construct the Maschke-type 
theorem for the BiHom-smash product $A \# H$. We also prove that $\left[A^{H}, A^{H} A_{A \# H}, A \# H A_{A^{H}}, A \# H\right]$ forms an associated Morita context, where $\left(A^{H},\left.\alpha_{A}\right|_{A^{H}},\left.\beta_{A}\right|_{A^{H}}\right)$ is a BiHom-subalgebra of $\left(H, \alpha_{H}, \beta_{H}\right)$-invariants in $\left(A, \alpha_{A}, \beta_{A}\right)$.

\section{Preliminaries}

We work over a base field $\mathbb{k}$. All algebras, linear spaces, etc. will be over $\mathbb{k}$; unadorned $\otimes$ means $\otimes_{\mathbb{k}}$. We use Sweedler's notation for terminologies on coalgebras. For a coalgebra $C$, we write comultiplication $\Delta(c)=c_{1} \otimes c_{2}$, for any $c \in C$.

Definition 1 ([7]). A BiHom-associative algebra is a 4-tuple $(A, \mu, \alpha, \beta)$, where $A$ is a linear space and $\alpha, \beta: \mathrm{A} \longrightarrow \mathrm{A}$ and $\mu: \mathrm{A} \otimes \mathrm{A} \longrightarrow \mathrm{A}$ are linear maps such that $\alpha \circ \beta=\beta \circ \alpha$, $\alpha(x \cdot y)=\alpha(x) \cdot \alpha(y), \beta(x \cdot y)=\beta(x) \cdot \beta(y)$ and

$$
\alpha(x) \cdot(y \cdot z)=(x \cdot y) \cdot \beta(z)
$$

for all $x, y, z \in A$. The maps $\alpha$ and $\beta$ (in this order) are called the structure maps of $A$, and condition (1) is called the BiHom-associativity condition.

A morphism $f:\left(A, \mu_{A}, \alpha_{A}, \beta_{A}\right) \longrightarrow\left(B, \mu_{B}, \alpha_{B}, \beta_{B}\right)$ of BiHom-associative algebras is a linear map $f: A \longrightarrow B$ such that $\alpha_{B} \circ f=f \circ \alpha_{A}, \beta_{B} \circ f=f \circ \beta_{A}$, and $f \circ \mu_{A}=\mu_{B} \circ(f \otimes f)$.

A BiHom-associative algebra $(A, \mu, \alpha, \beta)$ is called unital if there exists an element $1_{A} \in A$ (called a unit) such that $\alpha\left(1_{A}\right)=1_{A}, \beta\left(1_{A}\right)=1_{A}$ and

$$
a 1_{A}=\alpha(a) \text { and } 1_{A} a=\beta(a), \forall a \in A .
$$

Definition 2 ([7]). A BiHom-coassociative coalgebra is a 4tuple $(C, \Delta, \psi, \omega)$, in which $\mathrm{C}$ is a linear space, and $\psi, \omega: \mathrm{C}$ $\longrightarrow \mathrm{C}$ and $\Delta: \mathrm{C} \longrightarrow \mathrm{C} \otimes \mathrm{C}$ are linear maps, such that $\psi \circ$ $\omega=\omega \circ \psi,(\psi \otimes \psi) \circ \Delta=\Delta \circ \psi,(\omega \otimes \omega) \circ \Delta=\Delta \circ \omega$, and

$$
(\Delta \otimes \psi) \circ \Delta=(\omega \otimes \Delta) \circ \Delta
$$

The maps $\psi$ and $\omega$ (in this order) are called the structure maps of $C$, and condition (3) is called the BiHomcoassociativity condition.

Let us record the formula expressing the BiHomcoassociativity of $\Delta$ :

$$
\Delta\left(c_{1}\right) \otimes \psi\left(c_{2}\right)=\omega\left(c_{1}\right) \otimes \Delta\left(c_{2}\right), \forall c \in C
$$

A morphism $g:\left(C, \Delta_{C}, \psi_{C}, \omega_{C}\right) \longrightarrow\left(D, \Delta_{D}, \psi_{D}, \omega_{D}\right)$ of BiHom-coassociative coalgebras is a linear map $g: C \longrightarrow$ $D$ such that $\psi_{D} \circ g=g \circ \psi_{C}, \omega_{D} \circ g=g \circ \omega_{C}$, and $(g \otimes g) \circ$ $\Delta_{C}=\Delta_{D} \circ g$.

A BiHom-coassociative coalgebra $(C, \Delta, \psi, \omega)$ is called counital if there exists a linear map $\varepsilon: C \longrightarrow \mathbb{k}$ (called a counit) such that

$$
\begin{gathered}
\varepsilon \circ \psi=\varepsilon, \varepsilon \circ \omega=\varepsilon, \\
\left(i d_{C} \otimes \varepsilon\right) \circ \Delta=\omega \text { and }\left(\varepsilon \otimes i d_{C}\right) \circ \Delta=\psi .
\end{gathered}
$$

Definition 3 ([7]). A BiHom-bialgebra is a 7-tuple $(H, \mu, \Delta$, $\alpha, \beta, \psi, \omega)$, with the property that $(\mathrm{H}, \mu, \alpha, \beta)$ is a BiHomassociative algebra, $(H, \Delta, \psi, \omega)$ is a BiHom-coassociative coalgebra and moreover, the following relations are satisfied, for all $h, h^{\prime} \in H$ :

$$
\begin{aligned}
\Delta\left(h h^{\prime}\right) & =h_{1} h_{1}^{\prime} \otimes h_{2} h_{2}^{\prime}, \\
\alpha \circ \psi & =\psi \circ \alpha, \alpha \circ \omega=\omega \circ \alpha, \beta \circ \psi=\psi \circ \beta, \beta \circ \omega=\omega \circ \beta, \\
(\alpha \otimes \alpha) \circ \Delta & =\Delta \circ \alpha,(\beta \otimes \beta) \circ \Delta=\Delta \circ \beta, \\
\psi\left(h h^{\prime}\right) & =\psi(h) \psi\left(h^{\prime}\right), \omega\left(h h^{\prime}\right)=\omega(h) \omega\left(h^{\prime}\right) .
\end{aligned}
$$

We say that $H$ is a unital and counital BiHom-bialgebra if, in addition, it admits a unit $1_{H}$ and a counit $\varepsilon_{H}$ such that

$\Delta\left(1_{H}\right)=1_{H} \otimes 1_{H}, \varepsilon_{H}\left(1_{H}\right)=1, \psi\left(1_{H}\right)=1_{H}, \omega\left(1_{H}\right)=1_{H}$,

$\varepsilon_{H} \circ \alpha=\varepsilon_{H}, \varepsilon_{H} \circ \beta=\varepsilon_{H}, \varepsilon_{H}\left(h h^{\prime}\right)=\varepsilon_{H}(h) \varepsilon_{H}\left(h^{\prime}\right), \forall h, h^{\prime} \in H$.

Let $(H, \mu, \Delta, \alpha, \beta, \psi, \omega)$ be a unital and counital BiHombialgebra with a unit $1_{H}$ and a counit $\varepsilon_{H}$. A linear map $S: H$ $\longrightarrow H$ is called an antipode if it commutes with all the maps $\alpha, \beta, \psi, \omega$, and it satisfies the following relation:

$$
\beta \psi\left(S\left(h_{1}\right)\right) \alpha \omega\left(h_{2}\right)=\varepsilon_{H}(h) 1_{H}=\beta \psi\left(h_{1}\right) \alpha \omega\left(S\left(h_{2}\right)\right), \forall h \in H .
$$

A BiHom-Hopf algebra is a unital and counital BiHombialgebra with an antipode.

We can get some properties of the antipode. The proof is similar to the monoidal BiHom-Hopf algebra case in ([7], Proposition 6.6).

Proposition 4. Let $(H, \mu, \Delta, \alpha, \beta, \psi, \omega, S)$ be a BiHom-Hopf algebra. Then,

$$
\begin{gathered}
S\left(1_{H}\right)=1_{H}, \varepsilon_{H} \circ S=\varepsilon_{H}, \\
S(\beta(a) \alpha(b))=S(\beta(b)) S(\alpha(a)), \forall a, b \in H, \\
\psi\left(S(h)_{1}\right) \otimes \omega\left(S(h)_{2}\right)=\omega\left(S\left(h_{2}\right)\right) \otimes \psi\left(S\left(h_{1}\right)\right), \forall h \in H .
\end{gathered}
$$

Definition $5([7,10])$.

Let $\left(A, \mu_{A}, \alpha_{A}, \beta_{A}\right)$ be a BiHom-associative algebra and $\left(\mathrm{M}, \alpha_{\mathrm{M}}, \beta_{\mathrm{M}}\right)$ a triple where $M$ is a linear space, and $\alpha_{M}$, $\beta_{M}: M \longrightarrow$ Mare commuting linear maps.

(i) $\left(M, \alpha_{M}, \beta_{M}\right)$ is a left $A$-module if we have a linear map $A \otimes M \longrightarrow M, a \otimes m \mapsto a \cdot m$, such that $\alpha_{M}(a \cdot m)=$ $\alpha_{A}(a) \cdot \alpha_{M}(m), \beta_{M}(a \cdot m)=\beta_{A}(a) \cdot \beta_{M}(m)$, and

$\alpha_{A}(a) \cdot\left(a^{\prime} \cdot m\right)=\left(a \cdot a^{\prime}\right) \cdot \beta_{M}(m), \forall a, a^{\prime} \in A, m \in M$. 
If $\left(M, \alpha_{M}, \beta_{M}\right)$ and $\left(N, \alpha_{N}, \beta_{N}\right)$ are left $A$-modules (both $A$-actions denoted by .), a morphism of left $A$-modules $f: M \longrightarrow N$ is a linear map satisfying the conditions $\alpha_{N}$ $\circ f=f \circ \alpha_{M}, \beta_{N} \circ f=f \circ \beta_{M}$ and $f(a \cdot m)=a \cdot f(m)$, for all $a \in A$ and $m \in M$.

If $\left(A, \mu_{A}, \alpha_{A}, \beta_{A}, 1_{A}\right)$ is a unital BiHom-associative algebra and $\left(M, \alpha_{M}, \beta_{\mathrm{M}}\right)$ is a left $A$-module, then $M$ is called unital if $1_{A} \cdot m=\beta_{M}(m)$, for all $m \in M$.

(ii) $\left(M, \alpha_{M}, \beta_{M}\right)$ is a right $A$-module if we have a linear map $M \otimes A \longrightarrow M, m \otimes a \mapsto m \cdot a$, such that $\alpha_{M}(m$. $a)=\alpha_{M}(m) \cdot \alpha_{A}(a), \beta_{M}(m \cdot a)=\beta_{M}(m) \cdot \beta_{A}(a)$, and

$$
\alpha_{M}(m) \cdot\left(a \cdot a^{\prime}\right)=(m \cdot a) \cdot \beta_{A}\left(a^{\prime}\right), \forall a, a^{\prime} \in A, m \in M .
$$

If $\left(A, \mu_{A}, \alpha_{A}, \beta_{A}, 1_{A}\right)$ is a unital BiHom-associative algebra and $\left(M, \alpha_{M}, \beta_{M}\right)$ is a right $A$-module, then $M$ is called unital if $m \cdot 1_{A}=\alpha_{M}(m)$, for all $m \in M$.

(iii) If $\left(M, \alpha_{M}, \beta_{M}\right)$ is a left $\left(A, \alpha_{A}, \beta_{A}\right)$-module and a right $\left(B, \alpha_{B}, \beta_{B}\right)$-module, then $M$ is called an $(A, B)$ -bimodule if

$$
\alpha_{A}(a) \cdot\left(m \cdot a^{\prime}\right)=(a \cdot m) \cdot \beta_{B}\left(a^{\prime}\right), \forall a \in A, a^{\prime} \in B, m \in M .
$$

(iv) Let $\left(H, \mu_{H}, \Delta_{H}, \alpha_{H}, \beta_{H}, \psi_{H}, \omega_{H}\right)$ be a BiHombialgebra for which the maps $\alpha_{H}, \beta_{H}, \psi_{H}, \omega_{H}$ are bijective. A BiHom-associative algebra $\left(A, \mu_{A}, \alpha_{A}\right.$, $\left.\beta_{A}\right)$ is called a left $H$-module BiHom-algebra if $(A$, $\left.\alpha_{A}, \beta_{A}\right)$ is a left $\left(H, \alpha_{H}, \beta_{H}\right)$-module, with action denoted by $H \otimes A \longrightarrow A, h \otimes a \mapsto h \cdot a$, such that the following condition is satisfied:

$h \cdot\left(a a^{\prime}\right)=\left[\alpha_{H}^{-1} \omega_{H}^{-1}\left(h_{1}\right) \cdot a\right]\left[\beta_{H}^{-1} \psi_{H}^{-1}\left(h_{2}\right) \cdot a^{\prime}\right], \forall h \in H, a, a^{\prime} \in A$.

\section{The Maschke-Type Theorem for the BiHom- Smash Product $A \# H$}

In this section, we will give a Maschke-type theorem for the BiHom-smash product $\left(A \# H, \alpha_{A} \otimes \alpha_{H}, \beta_{A} \otimes \beta_{H}\right)$ over a semisimple BiHom-Hopf algebra $\left(H, \mu_{H}, \Delta_{H}, \alpha_{H}, \beta_{H}, \psi_{H}\right.$, $\left.\omega_{H}, S_{H}\right)$.

Definition 6 ([7]). Let $\left(H, \mu_{H}, \Delta_{H}, \alpha_{H}, \beta_{H}, \psi_{H}, \omega_{H}\right)$ be a BiHom-bialgebra and $\left(A, \mu_{A}, \alpha_{A}, \beta_{A}\right)$ a left $\mathrm{H}$-module BiHom-algebra, with the left action $H \otimes A \longrightarrow A, h \otimes a \mapsto h$ - $a$ such that all structure maps $\alpha_{H}, \beta_{H}, \psi_{H}, \omega_{H}, \alpha_{A}, \beta_{A}$ are bijective. The BiHom-smash product $\left(A \# H, \alpha_{A} \otimes \alpha_{H}, \beta_{A} \otimes\right.$
$\left.\beta_{H}\right)$ is defined on the vector space $A \otimes H$, and the BiHommultiplication is given by

$$
(a \# h)\left(a^{\prime} \# h^{\prime}\right)=a\left(\beta_{H}^{-1} \omega_{H}^{-1}\left(h_{1}\right) \cdot \beta_{A}^{-1}\left(a^{\prime}\right)\right) \# \psi_{H}^{-1}\left(h_{2}\right) h^{\prime},
$$

for all $a, a^{\prime} \in A, h, h^{\prime} \in H$. Note that $\left(A \# H, \alpha_{A} \otimes \alpha_{H}, \beta_{A} \otimes\right.$ $\left.\beta_{H}\right)$ is a BiHom-associative algebra. Moreover, if $A$ and $H$ are both unital with the units $1_{A}$ and $1_{H}$, then $\left(A \# H, \alpha_{A} \otimes\right.$ $\left.\alpha_{H}, \beta_{A} \otimes \beta_{H}\right)$ also has a unit $1_{A} \# 1_{H}$.

We assume that the BiHom-smash product in our paper is unital.

Proposition 7. Let $\left(A \# H, \alpha_{A} \otimes \alpha_{H}, \beta_{A} \otimes \beta_{H}\right)$ be a BiHomsmash product, then there are two BiHom-algebra isomorphisms $A \cong A \# 1_{H}$ via $a \mapsto a \# 1_{H}$ and $H \cong 1_{A} \# H$ via $h \mapsto 1_{A}$ $\# h$. This means $\left(a \# 1_{H}\right)\left(b \# 1_{H}\right)=a b \# 1_{H}$ and $\left(1_{A} \# h\right)\left(1_{A} \# g\right)$ $=1_{A} \# h g$, for all $a, b \in A$ and $h, g \in H$.

Proof. A straightforward computation left to the reader.

Our next result is the BiHom-analogue of the integral (for a Hom-analogue, see [17] and monoidal Hom-analogue, see [16]).

Definition 8. Let $\left(H, \mu_{H}, \Delta_{H}, \alpha_{H}, \beta_{H}, \psi_{H}, \omega_{H}, 1_{H}, \varepsilon_{H}, S_{H}\right)$ be a BiHom-Hopf algebra. A left integral in $H$ is an element $t \in H$ which is $\alpha_{H}$ and $\beta_{H}$-invariant (i.e., $\alpha_{H}(t)=t, \beta_{H}(t)=t$ ) such that

$$
h t=\varepsilon(h) t,
$$

for all $h \in H$. A left integral $t$ is normalized if $\varepsilon_{H}(t)=1$. Similarly, we can define right integrals in $H$. We denote the space of left and right integrals in $H$ by $\int_{l}^{H}$ and $\int_{r}^{H}$. If $\int_{l}^{H}=\int_{r}^{H}$, then we say $H$ is unimodular. $H$ is semisimple if and only if $H$ possesses a normalized left integral if and only if $H$ possesses a normalized right integral.

Proposition 9. Let $\left(H, \mu_{H}, \Delta_{H}, \alpha_{H}, \beta_{H}, \psi_{H}, \omega_{H}, 1_{H}, \varepsilon_{H}, S_{H}\right)$ be a finite-dimensional BiHom-Hopf algebra with $0 \neq t \in \int_{r}^{H}$, $\left(A, \alpha_{A}, \beta_{A}\right)$ be an $\left(H, \alpha_{H}, \beta_{H}\right)$-module BiHom-algebra, and $\left(M, \alpha_{M}, \beta_{M}\right),\left(N, \alpha_{N}, \beta_{N}\right)$, be left $\left(A \# H, \alpha_{A} \otimes \alpha_{H}, \beta_{A} \otimes \beta_{H}\right)$ -modules. If $\lambda:\left(M, \alpha_{M}, \beta_{M}\right) \longrightarrow\left(N, \alpha_{N}, \beta_{N}\right)$ is an $\left(A, \alpha_{A}\right.$, $\beta_{A}$ )-module map, then

$$
\begin{aligned}
\tilde{\lambda}:\left(M, \alpha_{M}, \beta_{M}\right) & \longrightarrow\left(N, \alpha_{N}, \beta_{N}\right), \\
m & \mapsto \beta_{H} \omega_{H}^{-1} S_{H}\left(t_{1}\right) \cdot \lambda\left(\psi_{H}^{-1}\left(t_{2}\right) \cdot \beta_{M}^{-2}(m)\right)
\end{aligned}
$$

is an $\left(A \# H, \alpha_{A} \otimes \alpha_{H}, \beta_{A} \otimes \beta_{H}\right)$-module morphism, where $\Delta_{H}(t)=t_{1} \otimes t_{2}$. 
Proof. First, show that $\alpha_{N} \circ \tilde{\lambda}=\tilde{\lambda} \circ \alpha_{M}$. For all $m \in M$,

$$
\begin{aligned}
\alpha_{N} \circ \tilde{\lambda}(m)= & \alpha_{N}(\tilde{\lambda}(m)) \\
= & \alpha_{H} \beta_{H} \omega_{H}^{-1} S_{H}\left(t_{1}\right) \cdot \alpha_{N} \lambda\left(\psi_{H}^{-1}\left(t_{2}\right) \cdot \beta_{M}^{-2}(m)\right) \\
= & \beta_{H} \omega_{H}^{-1} S_{H}\left(\alpha_{H}\left(t_{1}\right)\right) \cdot \lambda\left(\psi_{H}^{-1}\left(\alpha_{H}\left(t_{2}\right)\right) \cdot \alpha_{M} \beta_{M}^{-2}(m)\right) \\
= & \beta_{H} \omega_{H}^{-1} S_{H}\left(t_{1}\right) \cdot \lambda\left(\psi_{H}^{-1}\left(t_{2}\right) \cdot \beta_{M}^{-2}\left(\alpha_{M}(m)\right)\right) \\
& \cdot\left(\text { by } \alpha_{H}(t)=t\right) \\
= & \tilde{\lambda}\left(\alpha_{M}(m)\right) .
\end{aligned}
$$

Similarly, from $\beta_{H}(t)=t$, we get $\beta_{N}(\tilde{\lambda}(m))=\tilde{\lambda}\left(\beta_{M}(m)\right)$.

Since $\left(H, \alpha_{H}, \beta_{H}, \psi_{H}, \omega_{H}\right)$ is finite-dimensional and there is a nonzero integral $t$, it follows that $S_{H}$ is bijective. Meanwhile, all structure maps $\alpha_{H}, \beta_{H}, \psi_{H}, \omega_{H}, \alpha_{A}, \beta_{A}$ are bijective; thus, for $g \in H$, there exists an element $h \in H$ such that $g=$ $S_{H}\left(\alpha_{H} \beta_{H}^{3} \omega_{H} \psi_{H}^{2}(h)\right)$. For any $m \in M$, we have

$$
\begin{aligned}
& g \cdot \tilde{\lambda}(m)=S_{H}\left(\alpha_{H} \beta_{H}^{3} \omega_{H} \psi_{H}^{2}(h)\right) \cdot\left[\beta_{H} \omega_{H}^{-1} S_{H}\left(t_{1}\right)\right. \\
& \left.\cdot \lambda\left(\psi_{H}^{-1}\left(t_{2}\right) \cdot \beta_{M}^{-2}(m)\right)\right] \\
& \stackrel{(7)}{=}\left[S_{H}\left(\beta_{H}^{3} \omega_{H} \psi_{H}^{2}(h)\right) S_{H}\left(\beta_{H} \omega_{H}^{-1}\left(t_{1}\right)\right)\right] \\
& \cdot \lambda\left(\beta_{H} \psi_{H}^{-1}\left(t_{2}\right) \cdot \beta_{M}^{-1}(m)\right) \\
& =\left[S_{H} \beta_{H}\left(\beta_{H}^{2} \omega_{H} \psi_{H}^{2}(h)\right) S_{H} \alpha_{H}\left(\omega_{H}^{-1}\left(t_{1}\right)\right)\right] \\
& \cdot \lambda\left(\alpha_{H} \psi_{H}^{-1}\left(t_{2}\right) \cdot \beta_{M}^{-1}(m)\right)\left(\text { by } \alpha_{H}(t)=t\right) \\
& \stackrel{(75)}{=} S_{H}\left(\beta_{H} \omega_{H}^{-1}\left(t_{1}\right) \alpha_{H} \beta_{H}^{2} \omega_{H} \psi_{H}^{2}(h)\right) \cdot \lambda\left(\alpha_{H} \psi_{H}^{-1}\left(t_{2}\right) \cdot \beta_{M}^{-1}(m)\right) \\
& =S_{H}\left(\beta_{H} \omega_{H}^{-1}\left(t_{1}\right) \alpha_{H} \beta_{H}^{2} \psi_{H}^{2}\left(h_{1}\right) \varepsilon_{H}\left(h_{2}\right)\right) \\
& \cdot \lambda\left(\alpha_{H} \psi_{H}^{-1}\left(t_{2}\right) \cdot \beta_{M}^{-1}(m)\right) \\
& =S_{H}\left(\beta_{H} \omega_{H}^{-1}\left(t_{1}\right) \alpha_{H} \beta_{H}^{2} \psi_{H}^{2}\left(h_{1}\right)\right) \\
& \cdot \lambda\left(\left(\psi_{H}^{-1}\left(t_{2}\right) 1_{H} \varepsilon_{H}\left(h_{2}\right)\right) \cdot \beta_{M}^{-1}(m)\right) \\
& \stackrel{(753)}{=} S_{H}\left(\alpha_{H} \beta_{H} \omega_{H}^{-1}\left(t_{1}\right) \alpha_{H} \beta_{H}^{2} \psi_{H}^{2}\left(h_{1}\right)\right) \\
& \text { - } \lambda\left(\left(\alpha_{H} \psi_{H}^{-1}\left(t_{2}\right)\left(\beta_{H} \psi_{H}\left(h_{21}\right) \alpha_{H} \omega_{H} S_{H}\left(h_{22}\right)\right)\right) \cdot \beta_{M}^{-1}(m)\right) \\
& \stackrel{(7531)}{=} S_{H}\left(\alpha_{H} \beta_{H} \omega_{H}^{-1}\left(t_{1}\right) \alpha_{H} \beta_{H}^{2} \psi_{H}^{2}\left(h_{1}\right)\right) \\
& \cdot \lambda\left[\left(\left(\psi_{H}^{-1}\left(t_{2}\right) \beta_{H} \psi_{H}\left(h_{21}\right)\right) \alpha_{H} \beta_{H} \omega_{H} S_{H}\left(h_{22}\right)\right) \cdot \beta_{M}^{-1}(m)\right] \\
& \stackrel{(75312)}{=} S_{H}\left(\alpha_{H} \beta_{H} \omega_{H}^{-1}\left(t_{1}\right) \alpha_{H} \beta_{H}^{2} \omega_{H}^{-1} \psi_{H}^{2}\left(h_{11}\right)\right) \\
& \text { - } \lambda\left[\left(\left(\psi_{H}^{-1}\left(t_{2}\right) \beta_{H} \psi_{H}\left(h_{12}\right)\right) \alpha_{H} \beta_{H} \omega_{H} \psi_{H} S_{H}\left(h_{2}\right)\right) \cdot \beta_{M}^{-1}(m)\right] \\
& =S_{H} \alpha_{H} \beta_{H} \omega_{H}^{-1}\left(t_{1} \beta_{H} \psi_{H}^{2}\left(h_{11}\right)\right) \\
& \text { - } \lambda\left[\left(\psi_{H}^{-1}\left(t_{2} \beta_{H} \psi_{H}^{2}\left(h_{12}\right)\right) \alpha_{H} \beta_{H} \omega_{H} \psi_{H} S_{H}\left(h_{2}\right)\right) \cdot \beta_{M}^{-1}(m)\right] \\
& =S_{H} \alpha_{H} \beta_{H} \omega_{H}^{-1}\left(t_{1} \varepsilon_{H}\left(h_{1}\right)\right) \\
& \cdot \lambda\left[\left(\psi_{H}^{-1}\left(t_{2}\right) \alpha_{H} \beta_{H} \omega_{H} \psi_{H} S_{H}\left(h_{2}\right)\right) \cdot \beta_{M}^{-1}(m)\right] \\
& =S_{H} \alpha_{H} \beta_{H} \omega_{H}^{-1}\left(t_{1}\right) \cdot \lambda\left[\left(\psi_{H}^{-1}\left(t_{2}\right) \alpha_{H} \beta_{H} \omega_{H} \psi_{H}^{2} S_{H}(h)\right) \cdot \beta_{M}^{-1}(m)\right] \\
& =\beta_{H} \omega_{H}^{-1}\left(S_{H}\left(t_{1}\right)\right) \cdot \lambda\left[\left(\alpha_{H}^{-1} \psi_{H}^{-1}\left(t_{2}\right) \beta_{H}^{-2}(g)\right) \cdot \beta_{M}^{-1}(m)\right] \\
& \stackrel{(753127)}{=} \beta_{H} \omega_{H}^{-1}\left(S_{H}\left(t_{1}\right)\right) \cdot \lambda\left[\psi_{H}^{-1}\left(t_{2}\right) \cdot\left(\beta_{H}^{-2}(g) \cdot \beta_{M}^{-2}(m)\right)\right] \\
& =\beta_{H} \omega_{H}^{-1}\left(S_{H}\left(t_{1}\right)\right) \cdot \lambda\left[\psi_{H}^{-1}\left(t_{2}\right) \cdot \beta_{M}^{-2}(g \cdot m)\right]=\tilde{\lambda}(g \cdot m) \text {, }
\end{aligned}
$$

which implies that $\tilde{\lambda}$ is a left $\left(H, \alpha_{H}, \beta_{H}\right)$-module morphism. Furthermore, we have

$$
\begin{aligned}
& \left(1_{A} \# h\right)\left(a \# 1_{H}\right)=\omega_{H}^{-1}\left(h_{1}\right) \cdot a \# \alpha_{H} \psi_{H}^{-1}\left(h_{2}\right), \\
& \left(a \# 1_{H}\right)\left(1_{A} \# h\right)=\alpha_{A}(a) \# \beta_{H}(h),
\end{aligned}
$$

for all $a \in A, h \in H$. Meanwhile,

$$
a \# h=\left(1_{A} \# \alpha_{H}^{-1} \psi_{H}^{-1}\left(h_{2}\right)\right)\left(S_{H}^{-1}\left(\alpha_{H}^{-1} \beta_{H}^{-1} \omega_{H}^{-1}\left(h_{1}\right)\right) \cdot \beta_{A}^{-2}(a) \# 1_{H}\right)
$$

obtains by the following computation:

$$
\begin{aligned}
& \left(1_{A} \# \alpha_{H}^{-1} \psi_{H}^{-1}\left(h_{2}\right)\right)\left(S_{H}^{-1}\left(\alpha_{H}^{-1} \beta_{H}^{-1} \omega_{H}^{-1}\left(h_{1}\right)\right) \cdot \beta_{A}^{-2}(a) \# 1_{H}\right) \\
& \stackrel{(2)}{=}\left[\alpha_{H}^{-1} \psi_{H}^{-1} \omega_{H}^{-1}\left(h_{21}\right) \cdot\left(S_{H}^{-1} \alpha_{H}^{-1} \beta_{H}^{-1} \omega_{H}^{-1}\left(h_{1}\right) \cdot \beta_{A}^{-2}(a)\right)\right] \# \psi_{H}^{-2}\left(h_{22}\right) \\
& \stackrel{(27)}{=}\left[\alpha_{H}^{-2} \psi_{H}^{-1} \omega_{H}^{-1}\left(h_{21}\right) S_{H}^{-1} \alpha_{H}^{-1} \beta_{H}^{-1} \omega_{H}^{-1}\left(h_{1}\right)\right] \cdot \beta_{A}^{-1}(a) \# \psi_{H}^{-2}\left(h_{22}\right) \\
& \stackrel{(272)}{=}\left[\alpha_{H}^{-2} \psi_{H}^{-1} \omega_{H}^{-1}\left(h_{12}\right) S_{H}^{-1} \alpha_{H}^{-1} \beta_{H}^{-1} \omega_{H}^{-2}\left(h_{11}\right)\right] \cdot \beta_{A}^{-1}(a) \# \psi_{H}^{-1}\left(h_{2}\right) \\
& =\left[S_{H}^{-1} \beta_{H}\left(S_{H} \alpha_{H}^{-2} \beta_{H}^{-1} \psi_{H}^{-1} \omega_{H}^{-1}\left(h_{12}\right)\right) S_{H}^{-1} \alpha_{H}\left(\alpha_{H}^{-2} \beta_{H}^{-1} \omega_{H}^{-2}\left(h_{11}\right)\right)\right] \\
& \quad \cdot \beta_{A}^{-1}(a) \# \psi_{H}^{-1}\left(h_{2}\right) \\
& \stackrel{(2725)}{=} S_{H}^{-1}\left[\alpha_{H}^{-2} \omega_{H}^{-2}\left(h_{11}\right) S_{H} \alpha_{H}^{-1} \beta_{H}^{-1} \psi_{H}^{-1} \omega_{H}^{-1}\left(h_{12}\right)\right] \cdot \beta_{A}^{-1}(a) \# \psi_{H}^{-1}\left(h_{2}\right) \\
& =S_{H}^{-1}\left[\beta_{H} \psi_{H}\left(\alpha_{H}^{-2} \beta_{H}^{-1} \omega_{H}^{-2} \psi_{H}^{-1}\left(h_{11}\right)\right) S_{H} \alpha_{H} \omega_{H}\left(\alpha_{H}^{-1} \beta_{H}^{-2} \psi_{H}^{-1} \omega_{H}^{-2}\left(h_{12}\right)\right)\right] \\
& \quad \cdot \beta_{A}^{-1}(a) \# \psi_{H}^{-1}\left(h_{2}\right) \\
& \stackrel{(27253)}{=} S_{H}^{-1}\left(1_{H}\right) \cdot \beta_{A}^{-1}(a) \# \psi_{H}^{-1}\left(h_{2}\right) \varepsilon_{H}\left(h_{1}\right) \\
& \stackrel{(272534)}{=} 1_{H} \cdot \beta_{A}^{-1}(a) \# \psi_{H}^{-1}\left(\varepsilon_{H}\left(h_{1}\right) h_{2}\right)=a \# h,
\end{aligned}
$$

for all $a \in A, h \in H$. Next, we claim that $\tilde{\lambda}$ is a left $\left(A, \alpha_{A}, \beta_{A}\right)$ -module morphism. Indeed, for all $a \in A, m \in M$, we get

$$
\begin{aligned}
a \cdot \tilde{\lambda}(m)= & a \cdot\left[\beta_{H} \omega_{H}^{-1} S_{H}\left(t_{1}\right) \cdot \lambda\left(\psi_{H}^{-1}\left(t_{2}\right) \cdot \beta_{M}^{-2}(m)\right)\right] \\
= & \left(a \# 1_{H}\right) \cdot\left[\left(1_{A} \# \beta_{H} \omega_{H}^{-1} S_{H}\left(t_{1}\right)\right) \cdot \lambda\left(\psi_{H}^{-1}\left(t_{2}\right) \cdot \beta_{M}^{-2}(m)\right)\right] \\
= & {\left[\left(\alpha_{A}^{-1}(a) \# 1_{H}\right)\left(1_{A} \# \beta_{H} \omega_{H}^{-1} S_{H}\left(t_{1}\right)\right)\right] } \\
& \cdot \lambda\left(\beta_{H} \psi_{H}^{-1}\left(t_{2}\right) \cdot \beta_{M}^{-1}(m)\right) \\
\stackrel{(73)}{=} & \left(a \# \beta_{H}^{2} \omega_{H}^{-1} S_{H}\left(t_{1}\right)\right) \cdot \lambda\left(\beta_{H} \psi_{H}^{-1}\left(t_{2}\right) \cdot \beta_{M}^{-1}(m)\right) \\
= & \left(a \# \beta_{H} \omega_{H}^{-1} S_{H}\left(t_{1}\right)\right) \cdot \lambda\left(\psi_{H}^{-1}\left(t_{2}\right) \cdot \beta_{M}^{-1}(m)\right)\left(b y \beta_{H}(t)=t\right) \\
\stackrel{(734)}{=} & {\left[( 1 _ { A } \# \alpha _ { H } ^ { - 1 } \beta _ { H } \omega _ { H } ^ { - 1 } \psi _ { H } ^ { - 1 } ( S _ { H } ( t _ { 1 } ) _ { 2 } ) ) \left(S_{H}^{-1} \alpha_{H}^{-1} \omega_{H}^{-2}\left(S_{H}\left(t_{1}\right)_{1}\right)\right.\right.} \\
& \left.\left.\cdot \beta_{A}^{-2}(a) \# 1_{H}\right)\right] \cdot \lambda\left(\psi_{H}^{-1}\left(t_{2}\right) \cdot \beta_{M}^{-1}(m)\right) \\
\left({ }^{7346)}=\right. & {\left[( 1 _ { A } \# \alpha _ { H } ^ { - 1 } \beta _ { H } \omega _ { H } ^ { - 2 } S _ { H } ( t _ { 1 1 } ) ) \left(\alpha_{H}^{-1} \omega_{H}^{-1} \psi_{H}^{-1}\left(t_{12}\right)\right.\right.} \\
& \left.\left.\cdot \beta_{A}^{-2}(a) \# 1_{H}\right)\right] \cdot \lambda\left(\psi_{H}^{-1}\left(t_{2}\right) \cdot \beta_{M}^{-1}(m)\right) \\
= & {\left[\left(1_{A} \# \beta_{H} \omega_{H}^{-2} S_{H}\left(t_{11}\right)\right)\left(\omega_{H}^{-1} \psi_{H}^{-1}\left(t_{12}\right) \cdot \beta_{A}^{-2}(a) \# 1_{H}\right)\right] } \\
& \cdot \lambda\left(\alpha_{H} \psi_{H}^{-1}\left(t_{2}\right) \cdot \beta_{M}^{-1}(m)\right)\left(b y \alpha_{H}(t)=t\right) \\
(73462) & {\left[\left(1_{A} \# \beta_{H} \omega_{H}^{-1} S_{H}\left(t_{1}\right)\right)\left(\omega_{H}^{-1} \psi_{H}^{-1}\left(t_{21}\right) \cdot \beta_{A}^{-2}(a) \# 1_{H}\right)\right] } \\
& \cdot \lambda\left(\alpha_{H} \psi_{H}^{-2}\left(t_{22}\right) \cdot \beta_{M}^{-1}(m)\right)
\end{aligned}
$$




$$
\begin{aligned}
& \stackrel{(734627)}{=}\left(1_{A} \# \alpha_{H} \beta_{H} \omega_{H}^{-1} S_{H}\left(t_{1}\right)\right) \cdot\left[\left(\omega_{H}^{-1} \psi_{H}^{-1}\left(t_{21}\right) \cdot \beta_{A}^{-2}(a) \# 1_{H}\right)\right. \\
&\left.\cdot \lambda\left(\alpha_{H} \beta_{H}^{-1} \psi_{H}^{-2}\left(t_{22}\right) \cdot \beta_{M}^{-2}(m)\right)\right] \\
&=\left(1_{A} \# \alpha_{H} \beta_{H} \omega_{H}^{-1} S_{H}\left(t_{1}\right)\right) \cdot \lambda\left[\left(\omega_{H}^{-1} \psi_{H}^{-1}\left(t_{21}\right) \cdot \beta_{A}^{-2}(a) \# 1_{H}\right)\right. \\
&\left.\cdot \cdot\left(\left(1_{A} \# \alpha_{H} \beta_{H}^{-1} \psi_{H}^{-2}\left(t_{22}\right)\right) \cdot \beta_{M}^{-2}(m)\right)\right] \\
& \stackrel{(7346277)}{=}\left(1_{A} \# \alpha_{H} \beta_{H} \omega_{H}^{-1} S_{H}\left(t_{1}\right)\right) \cdot \lambda\left[\left(\left(\alpha_{H}^{-1} \omega_{H}^{-1} \psi_{H}^{-1}\left(t_{21}\right)\right.\right.\right. \\
&\left.\left.\left.\cdot \alpha_{A}^{-1} \beta_{A}^{-2}(a) \# 1_{H}\right)\left(1_{A} \# \alpha_{H} \beta_{H}^{-1} \psi_{H}^{-2}\left(t_{22}\right)\right)\right) \cdot \beta_{M}^{-1}(m)\right] \\
& \stackrel{(73462773)}{=}\left(1_{A} \# \alpha_{H} \beta_{H} \omega_{H}^{-1} S_{H}\left(t_{1}\right)\right) \cdot \lambda\left[\left(\omega_{H}^{-1} \psi_{H}^{-1}\left(t_{21}\right)\right.\right. \\
&\left.\left.\quad \cdot \beta_{A}^{-2}(a) \# \alpha_{H} \psi_{H}^{-2}\left(t_{22}\right)\right) \cdot \beta_{M}^{-1}(m)\right] \\
&=\left(1_{A} \# \alpha_{H} \beta_{H} \omega_{H}^{-1} S_{H}\left(t_{1}\right)\right) \cdot \lambda\left[\left(\omega_{H}^{-1}\left(\psi_{H}^{-1}\left(t_{21}\right)\right)\right.\right. \\
&\left.\left.\cdot \beta_{A}^{-2}(a) \# \alpha_{H} \psi_{H}^{-1}\left(\psi_{H}^{-1}\left(t_{22}\right)\right)\right) \cdot \beta_{M}^{-1}(m)\right] \\
&(734627732)\left(1_{A} \# \alpha_{H} \beta_{H} \omega_{H}^{-1} S_{H}\left(t_{1}\right)\right) \\
& \cdot \lambda\left[\left(\left(1_{A} \# \psi_{H}^{-1}\left(t_{2}\right)\right)\left(\beta_{A}^{-2}(a) \# 1_{H}\right) \cdot \beta_{M}^{-1}(m)\right]\right. \\
&(7346277327)\left(1_{A} \# \alpha_{H} \beta_{H} \omega_{H}^{-1} S_{H}\left(t_{1}\right)\right) \\
&= \cdot \lambda\left[\left(1_{A} \# \alpha_{H} \psi_{H}^{-1}\left(t_{2}\right) \cdot\left(\left(\beta_{A}^{-2}(a) \# 1_{H}\right) \cdot \beta_{M}^{-2}(m)\right)\right]\right. \\
&=\left(1_{A} \# \beta_{H} \omega_{H}^{-1} S_{H}\left(t_{1}\right)\right) \cdot \lambda\left[\left(1_{A} \# \psi_{H}^{-1}\left(t_{2}\right)\right)\right. \\
&\left.\cdot\left(\beta_{A}^{-2}(a) \cdot \beta_{M}^{-2}(m)\right)\right]\left(b y \alpha_{H}(t)=t\right) \\
&=\left.\beta_{1}\right) \cdot \lambda\left(\psi_{H}^{-1}\left(t_{2}\right) \cdot \beta_{M}^{-2}(a \cdot m)\right)=\tilde{\lambda}(a \cdot m) . \\
&=
\end{aligned}
$$

Thus, we get that $\tilde{\lambda}$ is a left $\left(A \# H, \alpha_{A} \otimes \alpha_{H}, \beta_{A} \otimes \beta_{H}\right)$ -module morphism.

Proposition 10. Let $\left(H, \mu_{H}, \Delta_{H}, \alpha_{H}, \beta_{H}, \psi_{H}, \omega_{H}, 1_{H}, \varepsilon_{H}, S_{H}\right)$ be a finite-dimensional semisimple BiHom-Hopf algebra and $\left(A, \alpha_{A}, \beta_{A}\right)$ be a left $\left(H, \alpha_{H}, \beta_{H}\right)$-module BiHom-algebra. Let $\left(M, \alpha_{M}, \beta_{M}\right)$ be a left $\left(A \# H, \alpha_{A} \otimes \alpha_{H}, \beta_{A} \otimes \beta_{H}\right)$-module and $\left(N, \alpha_{N}, \beta_{N}\right)$ an $\left(A \# H, \alpha_{A} \otimes \alpha_{H}, \beta_{A} \otimes \beta_{H}\right)$-submodule of $(M$, $\left.\alpha_{M}, \beta_{M}\right)$. If $\left(N, \alpha_{N}, \beta_{N}\right)$ is a direct summand of $\left(M, \alpha_{M}, \beta_{M}\right)$ as $\left(A, \alpha_{A}, \beta_{A}\right)$-modules, then $\left(N, \alpha_{N}, \beta_{N}\right)$ is also a direct summand of $\left(M, \alpha_{M}, \beta_{M}\right)$ as $\left(A \# H, \alpha_{A} \otimes \alpha_{H}, \beta_{A} \otimes \beta_{H}\right)$-modules.

Proof. Since $\left(H, \mu_{H}, \Delta_{H}, \alpha_{H}, \beta_{H}, \psi_{H}, \omega_{H}, 1_{H}, \varepsilon_{H}, S_{H}\right)$ is a finite-dimensional semisimple BiHom-Hopf algebra, there exists a normalized right integral $t$ in $H$. Let $\lambda:\left(M, \alpha_{M}, \beta_{M}\right)$ $\longrightarrow\left(N, \alpha_{N}, \beta_{N}\right)$ be a canonical projection as $\left(A, \alpha_{A}, \beta_{A}\right)$ -modules. Define

$$
\begin{aligned}
\tilde{\lambda}:\left(M, \alpha_{M}, \beta_{M}\right) & \longrightarrow\left(N, \alpha_{N}, \beta_{N}\right), \\
m & \mapsto \beta_{H} \omega_{H}^{-1} S_{H}\left(t_{1}\right) \cdot \lambda\left(\psi_{H}^{-1}\left(t_{2}\right) \cdot \beta_{M}^{-2}(m)\right) .
\end{aligned}
$$

Thus, by Proposition 9, we obtain that $\tilde{\lambda}$ is a left $(A \# H$, $\left.\alpha_{A} \otimes \alpha_{H}, \beta_{A} \otimes \beta_{H}\right)$-module morphism. Now, we show that $\tilde{\lambda}$ is also a projection. By the projrctivity of $\lambda$, we prove for all $n \in N$ that

$$
\begin{aligned}
\tilde{\lambda}(n) & =\beta_{H} \omega_{H}^{-1} S_{H}\left(t_{1}\right) \cdot \lambda\left(\psi_{H}^{-1}\left(t_{2}\right) \cdot \beta_{N}^{-2}(n)\right) \\
& =\beta_{H} \omega_{H}^{-1} S_{H}\left(t_{1}\right) \cdot\left(\psi_{H}^{-1}\left(t_{2}\right) \cdot \beta_{N}^{-2}(n)\right) \\
& \stackrel{(7)}{=}\left(\alpha_{H}^{-1} \beta_{H} \omega_{H}^{-1} S_{H}\left(t_{1}\right) \psi_{H}^{-1}\left(t_{2}\right)\right) \cdot \beta_{N}^{-1}(n) \\
& =\left(\beta_{H} \omega_{H}^{-1} S_{H}\left(t_{1}\right) \alpha_{H} \psi_{H}^{-1}\left(t_{2}\right)\right) \cdot \beta_{N}^{-1}(n)\left(b y \alpha_{H}(t)=t\right) \\
& =\omega_{H}^{-1} \psi_{H}^{-1}\left(\beta_{H} \psi_{H} S_{H}\left(t_{1}\right) \alpha_{H} \omega_{H}\left(t_{2}\right)\right) \cdot \beta_{N}^{-1}(n) \\
& \stackrel{(73)}{=} \varepsilon_{H}(t) 1_{H} \cdot \beta_{N}^{-1}(n)=1_{H} \cdot \beta_{N}^{-1}(n)=n .
\end{aligned}
$$

It follows $\left(N, \alpha_{N}, \beta_{N}\right)$ which is a direct summand of $(M$, $\left.\alpha_{M}, \beta_{M}\right)$ as left $\left(A \# H, \alpha_{A} \otimes \alpha_{H}, \beta_{A} \otimes \beta_{H}\right)$-modules.

By the above discussions, we obtain the Maschke-type theorem for BiHom-smash product, which generalizes Theorem 14 in [16].

Theorem 11. Let $\left(H, \mu_{H}, \Delta_{H}, \alpha_{H}, \beta_{H}, \psi_{H}, \omega_{H}, 1_{H}, \varepsilon_{H}, S_{H}\right)$ be a finite-dimensional semisimple BiHom-Hopf algebra and $\left(A, \alpha_{A}, \beta_{A}\right)$ be a left $\left(H, \alpha_{H}, \beta_{H}\right)$-module BiHom-algebra. If $\left(A, \alpha_{A}, \beta_{A}\right)$ is semisimple, then so is the BiHom-smash product $\left(A \# H, \alpha_{A} \otimes \alpha_{H}, \beta_{A} \otimes \beta_{H}\right)$.

\section{The Associated Morita Context}

The main aim of this section is to construct an associated Morita context between the BiHom-smash product $(A \# H$, $\left.\alpha_{A} \otimes \alpha_{H}, \beta_{A} \otimes \beta_{H}\right)$ and the BiHom-subalgebra $\left(A^{H}, \alpha_{A}\right.$ $\left.\left|A^{H}, \beta_{A}\right|_{A^{H}}\right)$. Note that the monoidal Hom-analogue of the Morita context has been studied in [16].

Let $\left(A, \alpha_{A}, \beta_{A}\right)$ be a left $\left(H, \alpha_{H}, \beta_{H}\right)$-module BiHomalgebra and

$$
A^{H}=\left\{a \in A \mid h \cdot a=\varepsilon(h) \beta_{A}(a), \forall h \in H\right\}
$$

be the $\left(H, \alpha_{H}, \beta_{H}\right)$-invariants of $\left(A, \alpha_{A}, \beta_{A}\right)$. It is easy to get $1_{A} \in A^{H}$. If $a, a^{\prime} \in A^{H}$, then both $\alpha_{A}(a), \beta_{A}(a)$ and $a a^{\prime}$ are in $A^{H}$. It follows $\left(A^{H},\left.\alpha_{A}\right|_{A^{H}},\left.\beta_{A}\right|_{A^{H}}\right)$ which is a BiHomsubalgebra of $\left(A, \alpha_{A}, \beta_{A}\right)$. For all $a, a^{\prime} \in A^{H}, h \in H$, we only check

$$
\begin{aligned}
h \cdot\left(\mathrm{a} a^{\prime}\right) & \stackrel{(10)}{=}\left[\alpha_{H}^{-1}\left(\omega_{H}^{-1}\left(h_{1}\right)\right) \cdot a\right]\left[\beta_{H}^{-1}\left(\psi_{H}^{-1}\left(h_{2}\right)\right) \cdot a^{\prime}\right] \\
& =\left(\varepsilon_{H}\left(h_{1}\right) \beta_{A}(a)\right)\left(\varepsilon_{H}\left(h_{2}\right) \beta_{A}\left(a^{\prime}\right)\right) \\
& =\varepsilon_{H}(h) \beta_{A}\left(a a^{\prime}\right) .
\end{aligned}
$$


Let $t$ be a normalized left integral of $\left(H, \alpha_{H}, \beta_{H}\right)$; then for all $h \in H, a \in A$, we have

$$
\begin{aligned}
h \cdot(t \cdot a) & \stackrel{(7)}{=}\left(\alpha_{H}^{-1}(h) t\right) \cdot \beta_{A}(a)=\varepsilon_{H}(h) t \cdot \beta_{A}(a) \\
& =\varepsilon_{H}(h) \beta_{H}(t) \cdot \beta_{A}(a)=\varepsilon_{H}(h) \beta_{H}(t \cdot a) .
\end{aligned}
$$

This means $t \cdot a \in A^{H}$. Conversely, if $a \in A^{H}$, then $\beta_{A}^{-1}$ (a) $\in A^{H}$ and $t \cdot \beta_{A}^{-1}(a)=\varepsilon_{H}(t) a=a$. So, $A^{H} \subset t \cdot A$. In summary, if $\left(H, \mu_{H}, \Delta_{H}, \alpha_{H}, \beta_{H}, \psi_{H}, \omega_{H}, 1_{H}, \varepsilon_{H}, S_{H}\right)$ is a BiHom-Hopf algebra with a normalized left integral $t$, then $t \cdot A=A^{H}$.

Lemma 12. Let $\left(H, \mu_{H}, \Delta_{H}, \alpha_{H}, \beta_{H}, \psi_{H}, \omega_{H}\right)$ be a BiHomHopf algebra with a bijective antipode $S_{H}$ and $\left(A, \alpha_{A}, \beta_{A}\right)$ be a left $\left(H, \alpha_{H}, \beta_{H}\right)$-module BiHom-algebra. Then, $\left(A, \alpha_{A}, \beta_{A}\right)$ is a left and right $\left(A \# H, \alpha_{A} \otimes \alpha_{H}, \beta_{A} \otimes \beta_{H}\right)$-module via the following module structure maps:

(i) $(a \# h) \longrightarrow b=a \beta_{A}^{-1}(h \cdot b)$,

(ii) $b \longleftarrow(a \# h)=S_{H}^{-1} \alpha_{H}^{-1} \beta_{H}(h) \cdot \beta_{A}^{-1}(b a)$,

for all $a, b \in A, h \in H$.

Proof. We prove the conditions (10) and (11) hold and leave the others to the reader. For (10),

$$
\begin{aligned}
& {[(a \# h)(b \# k)] \longrightarrow \beta_{A}(c) } \\
& \qquad \stackrel{(1)}{=}\left[a\left(\beta_{H}^{-1} \omega_{H}^{-1}\left(h_{1}\right) \cdot \beta_{A}^{-1}(b)\right) \# \psi_{H}^{-1}\left(h_{2}\right) k\right] \longrightarrow \beta_{A}(c) \\
&=\left(a\left(\beta_{H}^{-1} \omega_{H}^{-1}\left(h_{1}\right) \cdot \beta_{A}^{-1}(b)\right)\right) \beta_{A}^{-1}\left(\left(\psi_{H}^{-1}\left(h_{2}\right) k\right) \cdot \beta_{A}(c)\right) \\
&=\left(a\left(\beta_{H}^{-1} \omega_{H}^{-1}\left(h_{1}\right) \cdot \beta_{A}^{-1}(b)\right)\right)\left(\left(\beta_{H}^{-1} \psi_{H}^{-1}\left(h_{2}\right) \beta_{H}^{-1}(k)\right) \cdot c\right) \\
& \stackrel{(17)}{=} \alpha_{A}(a)\left[\left(\beta_{H}^{-1} \omega_{H}^{-1}\left(h_{1}\right) \cdot \beta_{A}^{-1}(b)\right)\right. \\
&\left.\times\left(\left(\beta_{H}^{-2} \psi_{H}^{-1}\left(h_{2}\right) \beta_{H}^{-2}(k)\right) \cdot \beta_{A}^{-1}(c)\right)\right] \\
&(\stackrel{177)}{=} \alpha_{A}(a)\left[( \beta _ { H } ^ { - 1 } \omega _ { H } ^ { - 1 } ( h _ { 1 } ) \cdot \beta _ { A } ^ { - 1 } ( b ) ) \left(\alpha_{H} \beta_{H}^{-2} \psi_{H}^{-1}\left(h_{2}\right)\right.\right. \\
&\left.\left.\cdot\left(\beta_{H}^{-2}(k) \cdot \beta_{A}^{-2}(c)\right)\right)\right] \\
&= \alpha_{A}(a)\left[( \alpha _ { H } ^ { - 1 } \omega _ { H } ^ { - 1 } ( \alpha _ { H } \beta _ { H } ^ { - 1 } ( h _ { 1 } ) ) \cdot \beta _ { A } ^ { - 1 } ( b ) ) \left(\beta_{H}^{-1} \psi_{H}^{-1}\left(\alpha_{H} \beta_{H}^{-1}\left(h_{2}\right)\right)\right.\right. \\
&\left.\left.\cdot\left(\beta_{H}^{-2}(k) \cdot \beta_{A}^{-2}(c)\right)\right)\right] \\
&(17710) \alpha_{A}(a)\left[\alpha_{H} \beta_{H}^{-1}(h) \cdot\left(\beta_{A}^{-1}(b)\left(\beta_{H}^{-2}(k) \cdot \beta_{A}^{-2}(c)\right)\right)\right] \\
&= \alpha_{A}(a) \beta_{A}^{-1}\left[\alpha_{H}(h) \cdot\left(b \beta_{A}^{-1}(k \cdot c)\right)\right] \\
&=\left(\alpha_{A}(a) \# \alpha_{H}(h)\right) \longrightarrow\left(b \beta_{A}^{-1}(k \cdot c)\right) \\
&=\left(\alpha_{A}(a) \# \alpha_{H}(h)\right) \longrightarrow((b \# k) \longrightarrow c) .
\end{aligned}
$$

For (11),

$$
\begin{aligned}
& \alpha_{A}(a) \longleftarrow((b \# h)(c \# k)) \\
& \stackrel{(1)}{=} \alpha_{A}(a) \longleftarrow\left[b\left(\beta_{H}^{-1} \omega_{H}^{-1}\left(h_{1}\right) \cdot \beta_{A}^{-1}(c)\right) \# \psi_{H}^{-1}\left(h_{2}\right) k\right] \\
& =S_{H}^{-1} \alpha_{H}^{-1} \beta_{H}\left(\psi_{H}^{-1}\left(h_{2}\right) k\right) \cdot \beta_{A}^{-1}\left[\alpha_{A}(a)\left(b\left(\beta_{H}^{-1} \omega_{H}^{-1}\left(h_{1}\right) \cdot \beta_{A}^{-1}(c)\right)\right)\right] \\
& \stackrel{(17)}{=} \alpha_{H}^{-1} \beta_{H} S_{H}^{-1}\left(\beta_{H}\left(\beta_{H}^{-1} \psi_{H}^{-1}\left(h_{2}\right)\right) \alpha_{H}\left(\alpha_{H}^{-1}(k)\right)\right) \\
& \cdot \beta_{A}^{-1}\left((a b)\left(\omega_{H}^{-1}\left(h_{1}\right) \cdot c\right)\right) \\
& \stackrel{(175)}{=}\left[\alpha_{H}^{-1} \beta_{H} S_{H}^{-1} \beta_{H}\left(\alpha_{H}^{-1}(k)\right) \alpha_{H}^{-1} \beta_{H} S_{H}^{-1} \alpha_{H}\left(\beta_{H}^{-1} \psi_{H}^{-1}\left(h_{2}\right)\right)\right] \\
& \cdot \beta_{A}^{-1}\left((a b)\left(\omega_{H}^{-1}\left(h_{1}\right) \cdot c\right)\right) \\
& =\left[\alpha_{H}^{-2} \beta_{H}^{2} S_{H}^{-1}(k) S_{H}^{-1} \psi_{H}^{-1}\left(h_{2}\right)\right] \cdot \beta_{A}^{-1}\left((a b)\left(\omega_{H}^{-1}\left(h_{1}\right) \cdot c\right)\right) \\
& \stackrel{(1757)}{=} \alpha_{H}^{-1} \beta_{H}^{2} S_{H}^{-1}(k) \cdot\left[S_{H}^{-1} \psi_{H}^{-1}\left(h_{2}\right) \cdot \beta_{A}^{-2}\left((a b)\left(\omega_{H}^{-1}\left(h_{1}\right) \cdot c\right)\right)\right] \\
& \stackrel{(175710)}{=} \alpha_{H}^{-1} \beta_{H}^{2} S_{H}^{-1}(k) \cdot\left[\left(\alpha_{H}^{-1} \omega_{H}^{-1}\left(\psi_{H}^{-1} S_{H}^{-1}\left(h_{2}\right)_{1}\right) \cdot \beta_{A}^{-2}(a b)\right)\right. \\
& \left.\times\left(\beta_{H}^{-1} \psi_{H}^{-1}\left(\psi_{H}^{-1} S_{H}^{-1}\left(h_{2}\right)_{2}\right) \cdot\left(\beta_{H}^{-2} \omega_{H}^{-1}\left(h_{1}\right) \cdot \beta_{A}^{-2}(c)\right)\right)\right] \\
& \stackrel{(1757106)}{=} \alpha_{H}^{-1} \beta_{H}^{2} S_{H}^{-1}(k) \cdot\left[\left(\alpha_{H}^{-1} \psi_{H}^{-2} S_{H}^{-1}\left(h_{22}\right) \cdot \beta_{A}^{-2}(a b)\right)\right. \\
& \left.\times\left(\beta_{H}^{-1} \omega_{H}^{-1} \psi_{H}^{-1} S_{H}^{-1}\left(h_{21}\right) \cdot\left(\beta_{H}^{-2} \omega_{H}^{-1}\left(h_{1}\right) \cdot \beta_{A}^{-2}(c)\right)\right)\right] \\
& \stackrel{(17571067)}{=} \alpha_{H}^{-1} \beta_{H}^{2} S_{H}^{-1}(k) \cdot\left[\left(\alpha_{H}^{-1} \psi_{H}^{-2} S_{H}^{-1}\left(h_{22}\right) \cdot \beta_{A}^{-2}(a b)\right)\right. \\
& \left.\times\left(\left(\alpha_{H}^{-1} \beta_{H}^{-1} \omega_{H}^{-1} \psi_{H}^{-1} S_{H}^{-1}\left(h_{21}\right) \beta_{H}^{-2} \omega_{H}^{-1}\left(h_{1}\right)\right) \cdot \beta_{A}^{-1}(c)\right)\right] \\
& \stackrel{(175710672)}{=} \alpha_{H}^{-1} \beta_{H}^{2} S_{H}^{-1}(k) \cdot\left[\left(\alpha_{H}^{-1} \psi_{H}^{-1} S_{H}^{-1}\left(h_{2}\right) \cdot \beta_{A}^{-2}(a b)\right)\right. \\
& \left.\times\left(\left(\alpha_{H}^{-1} \beta_{H}^{-1} \omega_{H}^{-1} \psi_{H}^{-1} S_{H}^{-1}\left(h_{12}\right) \beta_{H}^{-2} \omega_{H}^{-2}\left(h_{11}\right)\right) \cdot \beta_{A}^{-1}(c)\right)\right] \\
& =\alpha_{H}^{-1} \beta_{H}^{2} S_{H}^{-1}(k) \cdot\left[\left(\alpha_{H}^{-1} \psi_{H}^{-1} S_{H}^{-1}\left(h_{2}\right)\right) \cdot \beta_{A}^{-2}(a b)\right) \\
& \times\left(\left(S_{H}^{-1} \beta_{H}\left(\alpha_{H}^{-1} \beta_{H}^{-2} \omega_{H}^{-1} \psi_{H}^{-1}\left(h_{12}\right)\right) S_{H}^{-1} \alpha_{H}\right.\right. \\
& \left.\left.\left.\times\left(\alpha_{H}^{-1} \beta_{H}^{-2} \omega_{H}^{-2} S_{H}\left(h_{11}\right)\right)\right) \cdot \beta_{A}^{-1}(c)\right)\right] \\
& \stackrel{(1757106725)}{=} \alpha_{H}^{-1} \beta_{H}^{2} S_{H}^{-1}(k) \cdot\left[\left(\alpha_{H}^{-1} \psi_{H}^{-1} S_{H}^{-1}\left(h_{2}\right)\right) \cdot \beta_{A}^{-2}(a b)\right) \\
& \left.\times\left(S_{H}^{-1}\left(\alpha_{H}^{-1} \beta_{H}^{-1} \omega_{H}^{-2} S_{H}\left(h_{11}\right) \beta_{H}^{-2} \omega_{H}^{-1} \psi_{H}^{-1}\left(h_{12}\right)\right) \cdot \beta_{A}^{-1}(c)\right)\right] \\
& =\alpha_{H}^{-1} \beta_{H}^{2} S_{H}^{-1}(k) \cdot\left[\left(\alpha_{H}^{-1} \psi_{H}^{-1} S_{H}^{-1}\left(h_{2}\right)\right) \cdot \beta_{A}^{-2}(a b)\right) \\
& \times\left(S _ { H } ^ { - 1 } \left(\beta_{H} \psi_{H}\left(\alpha_{H}^{-1} \beta_{H}^{-2} \omega_{H}^{-2} \psi_{H}^{-1} S_{H}\left(h_{11}\right)\right) \alpha_{H} \omega_{H}\right.\right. \\
& \left.\left.\left.\times\left(\alpha_{H}^{-1} \beta_{H}^{-2} \omega_{H}^{-2} \psi_{H}^{-1}\left(h_{12}\right)\right)\right) \cdot \beta_{A}^{-1}(c)\right)\right] \\
& \stackrel{(17571067253)}{=} \alpha_{H}^{-1} \beta_{H}^{2} S_{H}^{-1}(k) \cdot\left[\left(\alpha_{H}^{-1} \psi_{H}^{-1} S_{H}^{-1}\left(h_{2}\right) \cdot \beta_{A}^{-2}(a b)\right) \varepsilon_{H}\left(h_{1}\right)\right. \\
& \left.\times\left(1_{H} \cdot \beta_{A}^{-1}(c)\right)\right] \\
& =\alpha_{H}^{-1} \beta_{H}^{2} S_{H}^{-1}(k) \cdot\left(\left(\alpha_{H}^{-1} S_{H}^{-1}(h) \cdot \beta_{A}^{-2}(a b)\right) c\right) \\
& =S_{H}^{-1} \alpha_{H}^{-1} \beta_{H}\left(\beta_{H}(k)\right) \cdot \beta_{A}^{-1}\left(\left(\alpha_{H}^{-1} S_{H}^{-1} \beta_{H}(h) \cdot \beta_{A}^{-1}(a b)\right) \beta_{A}(c)\right) \\
& =\left(S_{H}^{-1} \alpha_{H}^{-1} \beta_{H}(h) \cdot \beta_{A}^{-1}(a b)\right) \longleftarrow\left(\beta_{A}(c) \# \beta_{H}(k)\right) \\
& =(a \longleftarrow(b \# h)) \longleftarrow\left(\beta_{A}(c) \# \beta_{H}(k)\right) \text {, }
\end{aligned}
$$

for all $a, b, c \in A$ and $h, k \in H$.

Lemma 13. Let $\left(H, \mu_{H}, \Delta_{H}, \alpha_{H}, \beta_{H}, \psi_{H}, \omega_{H}\right)$ be a BiHomHopf algebra with a bijective antipode $S_{H}$ and $\left(A, \alpha_{A}, \beta_{A}\right)$ be a left $\left(H, \alpha_{H}, \beta_{H}\right)$-module BiHom-algebra. From Lemma 12, $\left(A, \alpha_{A}, \beta_{A}\right)$ is a left and right $\left(A \# H, \alpha_{A} \otimes \alpha_{H}, \beta_{A} \otimes \beta_{H}\right)$-module. Meanwhile, it is also a left and right $\left(A^{H},\left.\alpha_{A}\right|_{A^{H}},\left.\beta_{A}\right|_{A^{H}}\right)$ 
-module with the BiHom-multiplication. Thus, there are two bimodules $\left({ }_{A^{H}} A_{A \# H}, \alpha_{A}, \beta_{A}\right)$ and $\left({ }_{A \# H} A_{A^{H}}, \alpha_{A}, \beta_{A}\right)$.

Proof. By the BiHom-associativity, it is easy to get $\left(A, \alpha_{A}, \beta_{A}\right)$ which is a left and right $\left(A^{H},\left.\alpha_{A}\right|_{A^{H}},\left.\beta_{A}\right|_{A^{H}}\right)$-module. Now, we check the condition (12) hold for $\left({ }_{A^{H}} A_{A \# H}, \alpha_{A}, \beta_{A}\right)$ and $\left({ }_{A \# H} A_{A^{H}}, \alpha_{A}, \beta_{A}\right)$ as follows:

$$
\begin{aligned}
& (a b) \longleftarrow\left(\beta_{A}(c) \# \beta_{H}(h)\right) \\
& =S_{H}^{-1} \alpha_{H}^{-1} \beta_{H}^{2}(h) \cdot \beta_{A}^{-1}\left((a b) \beta_{A}(c)\right) \\
& \stackrel{(7)}{=} S_{H}^{-1} \alpha_{H}^{-1} \beta_{H}^{2}(h) \cdot \beta_{A}^{-1}\left(\alpha_{A}(a)(b c)\right) \\
& =S_{H}^{-1} \alpha_{H}^{-1} \beta_{H}^{2}(h) \cdot\left(\alpha_{A} \beta_{A}^{-1}(a) \beta_{A}^{-1}(b c)\right) \\
& \stackrel{(710)}{=}\left[\alpha_{H}^{-1} \omega_{H}^{-1}\left(S_{H}^{-1} \alpha_{H}^{-1} \beta_{H}^{2}(h)_{1}\right) \cdot \alpha_{A} \beta_{A}^{-1}(a)\right] \\
& \times\left[\beta_{H}^{-1} \psi_{H}^{-1}\left(S_{H}^{-1} \alpha_{H}^{-1} \beta_{H}^{2}(h)_{2}\right) \cdot \beta_{A}^{-1}(b c)\right] \\
& \stackrel{(7106)}{=}\left[\alpha_{H}^{-2} \beta_{H}^{-2} \psi_{H}^{-1} S_{H}^{-1}\left(h_{2}\right) \cdot \alpha_{A} \beta_{A}^{-1}(a)\right] \\
& \times\left[\alpha_{H}^{-1} \beta_{H} \omega_{H}^{-1} S_{H}^{-1}\left(h_{1}\right) \cdot \beta_{A}^{-1}(b c)\right] \\
& =\varepsilon_{H}\left(h_{2}\right) \alpha_{A}(a)\left[\alpha_{H}^{-1} \beta_{H} \omega_{H}^{-1} S_{H}^{-1}\left(h_{1}\right) \cdot \beta_{A}^{-1}(b c)\right] \\
& =\alpha_{A}(a)\left[S_{H}^{-1} \alpha_{H}^{-1} \beta_{H}(h) \cdot \beta_{A}^{-1}(b c)\right]=\alpha_{A}(a)(b \longleftarrow(c \# h)) \text {, } \\
& \left(\alpha_{A}(c) \# \alpha_{H}(h)\right) \longrightarrow(b a) \\
& =\alpha_{A}(c) \beta_{A}^{-1}\left(\alpha_{H}(h) \cdot(b a)\right) \\
& \stackrel{(10)}{=} \alpha_{A}(c) \beta_{A}^{-1}\left(\left(\omega_{H}^{-1}\left(h_{1}\right) \cdot b\right)\left(\alpha_{H} \beta_{H}^{-1} \psi_{H}^{-1}\left(h_{2}\right) \cdot a\right)\right) \\
& =\alpha_{A}(c) \beta_{A}^{-1}\left(\left(\omega_{H}^{-1}\left(h_{1}\right) \cdot b\right) \varepsilon_{H}\left(h_{2}\right) \beta_{A}(a)\right) \\
& =\alpha_{A}(c)\left(\beta_{A}^{-1}(h \cdot b) a\right) \\
& \stackrel{(107)}{=}\left(c \beta_{A}^{-1}(h \cdot b)\right) \beta_{A}(a)=((c \# h) \longrightarrow b) \beta_{A}(a),
\end{aligned}
$$

for all $a \in A^{H}, b, c \in A$ and $h \in H$.

Let $t$ be a left integral in $\left(H, \alpha_{H}, \beta_{H}, \psi_{H}, \omega_{H}\right)$. If $S_{H}(t)=t$, then $t$ is also a right integral in $\left(H, \alpha_{H}, \beta_{H}, \psi_{H}, \omega_{H}\right)$. For all $t \in H$,

$$
\begin{aligned}
t h & =S_{H}(t) S_{H}\left(S_{H}^{-1}(h)\right) \\
& =S_{H} \beta_{H}(t) S_{H} \alpha_{H}\left(\alpha_{H}^{-1} S_{H}^{-1}(h)\right) \\
& \stackrel{(5)}{=} S_{H} \beta_{H}\left(\alpha_{H}^{-1} S_{H}^{-1}(h)\right) S_{H} \alpha_{H}(t) \\
& =\alpha_{H}^{-1} \beta_{H}(h) S_{H}(t)=\alpha_{H}^{-1} \beta_{H}(h) t=\varepsilon_{H}(h) t .
\end{aligned}
$$

For any $a \in A, h \in H$,

$$
\begin{aligned}
& \left(1_{A} \# t\right)(a \# h) \\
& \stackrel{(4)}{=}\left(1_{A} \# t\right)\left[\left(1_{A} \# \alpha_{H}^{-1} \psi_{H}^{-1}\left(h_{2}\right)\right)\left(S_{H}^{-1} \alpha_{H}^{-1} \beta_{H}^{-1} \omega_{H}^{-1}\left(h_{1}\right) \cdot \beta_{A}^{-2}(a) \# 1_{H}\right)\right] \\
& \stackrel{(47)}{=}\left[\left(1_{A} \# t\right)\left(1_{A} \# \alpha_{H}^{-1} \psi_{H}^{-1}\left(h_{2}\right)\right)\right]\left(S_{H}^{-1} \alpha_{H}^{-1} \omega_{H}^{-1}\left(h_{1}\right) \cdot \beta_{A}^{-1}(a) \# 1_{H}\right) \\
& \quad=\left(1_{A} \# t \alpha_{H}^{-1} \psi_{H}^{-1}\left(h_{2}\right)\right)\left(S_{H}^{-1} \alpha_{H}^{-1} \omega_{H}^{-1}\left(h_{1}\right) \cdot \beta_{A}^{-1}(a) \# 1_{H}\right) \\
& \quad=\left(1_{A} \# t \varepsilon_{H}\left(h_{2}\right)\right)\left(S_{H}^{-1} \alpha_{H}^{-1} \omega_{H}^{-1}\left(h_{1}\right) \cdot \beta_{A}^{-1}(a) \# 1_{H}\right) \\
& \quad=\left(1_{A} \# t\right)\left(S_{H}^{-1} \alpha_{H}^{-1}(h) \cdot \beta_{A}^{-1}(a) \# 1_{H}\right) .
\end{aligned}
$$

So, we obtain

$$
\left(1_{A} \# t\right)(a \# h)=\left(1_{A} \# t\right)\left(S_{H}^{-1} \alpha_{H}^{-1}(h) \cdot \beta_{A}^{-1}(a) \# 1_{H}\right) .
$$

Now, with the above preparations, we can construct the associated Morita context between the BiHom-smash product $A \# H$ and the BiHom-subalgebra $A^{H}$.

Theorem 14. Let $\left(H, \alpha_{H}, \beta_{H}, \psi_{H}, \omega_{H}\right)$ be a BiHom-Hopf algebra with a bijective antipode $S_{H}$, $t$ be a left integral in $H$ satisfying $S_{H}(t)=t$, and $\left(A, \alpha_{A}, \beta_{A}\right)$ be a left $\left(H, \alpha_{H}, \beta_{H}\right)$-module BiHom-algebra. Then, $\left[A^{H},{ }_{A}{ }^{H} A_{A \# H},{ }_{A \# H} A_{A^{H}}, A \# H\right]$ forms an associated Morita context with the maps

$$
\begin{aligned}
{[-,-]:\left(A \otimes_{A^{H}} A, \alpha_{A} \otimes \alpha_{A}, \beta_{A} \otimes \beta_{A}\right) } & \longrightarrow\left(A \# H, \alpha_{A} \otimes \alpha_{H}, \beta_{A} \otimes \beta_{H}\right), \\
v i a[a, b] & =(a \# t)\left(b \# 1_{H}\right), \\
(-,-):\left(A \otimes_{A \# H} A, \alpha_{A} \otimes \alpha_{A}, \beta_{A} \otimes \beta_{A}\right) & \longrightarrow\left(A^{H},\left.\alpha_{A}\right|_{A^{H}},\left.\beta_{A}\right|_{A^{H}}\right), \\
\operatorname{via}(a, b) & =t \cdot \beta_{A}^{-1}(a b),
\end{aligned}
$$

for all $a, b \in A$.

Proof. We first prove $[-,-]$ is both an $\left(A \# H, \alpha_{A} \otimes \alpha_{H}\right.$, $\left.\beta_{A} \otimes \beta_{H}\right)$-module map and middle $\left(A^{H},\left.\alpha_{A}\right|_{A^{H}},\left.\beta_{A}\right|_{A^{H}}\right)$-linear map, which means $[-,-]$ satisfies the following three conditions:

$$
\begin{aligned}
\left(\alpha_{A}(a) \# \alpha_{H}(h)\right)[b, c] & =\left[(a \# h) \longrightarrow b, \beta_{A}(c)\right], \\
{[a, b]\left(\beta_{A}(c) \# \beta_{H}(h)\right) } & =\left[\alpha_{A}(a), b \longleftarrow(c \# h)\right], \\
{\left[a a^{\prime}, \beta_{A}(b)\right] } & =\left[\alpha_{A}(a), a^{\prime} b\right],
\end{aligned}
$$

for all $a, b, c \in A, h \in H$ and $a^{\prime} \in A^{H}$.

To prove these conditions, we compute

$$
\begin{aligned}
\left(\alpha_{A}(a) \# \alpha_{H}(h)\right)[b, c] & =\left(\alpha_{A}(a) \# \alpha_{H}(h)\right)\left[(b \# t)\left(c \# 1_{H}\right)\right] \\
\stackrel{(1)}{=} & {[(a \# h)(b \# t)]\left(\beta_{A}(c) \# 1_{H}\right) } \\
\stackrel{(13)}{=} & {\left[\left(\left(\alpha_{A}^{-1}(a) \# 1_{H}\right)\left(1_{A} \# \beta_{H}^{-1}(h)\right)\right)\left(\left(\alpha_{A}^{-1}(b) \# 1_{H}\right)\left(1_{A} \# t\right)\right)\right] } \\
& \times\left(\beta_{A}(c) \# 1_{H}\right) \\
\stackrel{(131)}{=} & {\left[\left(a \# 1_{H}\right)\left(\left(1_{A} \# \beta_{H}^{-1}(h)\right)\left(\left(\alpha_{A}^{-1} \beta_{A}^{-1}(b) \# 1_{H}\right)\left(1_{A} \# t\right)\right)\right)\right] } \\
& \times\left(\beta_{A}(c) \# 1_{H}\right) \\
\stackrel{(1311)}{=} & {\left[\left(a \# 1_{H}\right)\left(\left(\left(1_{A} \# \alpha_{H}^{-1} \beta_{H}^{-1}(h)\right)\left(\alpha_{A}^{-1} \beta_{A}^{-1}(b) \# 1_{H}\right)\right)\left(1_{A} \# t\right)\right)\right] } \\
& \times\left(\beta_{A}(c) \# 1_{H}\right) \\
\stackrel{(13112)}{=} & {\left[( a \# 1 _ { H } ) \left(\left(\alpha_{H}^{-1} \beta_{H}^{-1} \omega_{H}^{-1}\left(h_{1}\right) \cdot \alpha_{A}^{-1} \beta_{A}^{-1}(b) \# \beta_{H}^{-1} \psi_{H}^{-1}\left(h_{2}\right)\right)\right.\right.} \\
& \left.\left.\times\left(1_{A} \# t\right)\right)\right]\left(\beta_{A}(c) \# 1_{H}\right) \\
(131123) & {\left[( a \# 1 _ { H } ) \left(\left(\left(\alpha_{H}^{-2} \beta_{H}^{-1} \omega_{H}^{-1}\left(h_{1}\right) \cdot \alpha_{\mathrm{A}}^{-2} \beta_{A}^{-1}(b) \# 1_{H}\right)\right.\right.\right.} \\
& \left.\left.\left.\times\left(1_{A} \# \beta_{H}^{-2} \psi_{H}^{-1}\left(h_{2}\right)\right)\right)\left(1_{A} \# t\right)\right)\right]\left(\beta_{A}(c) \# 1_{H}\right)
\end{aligned}
$$




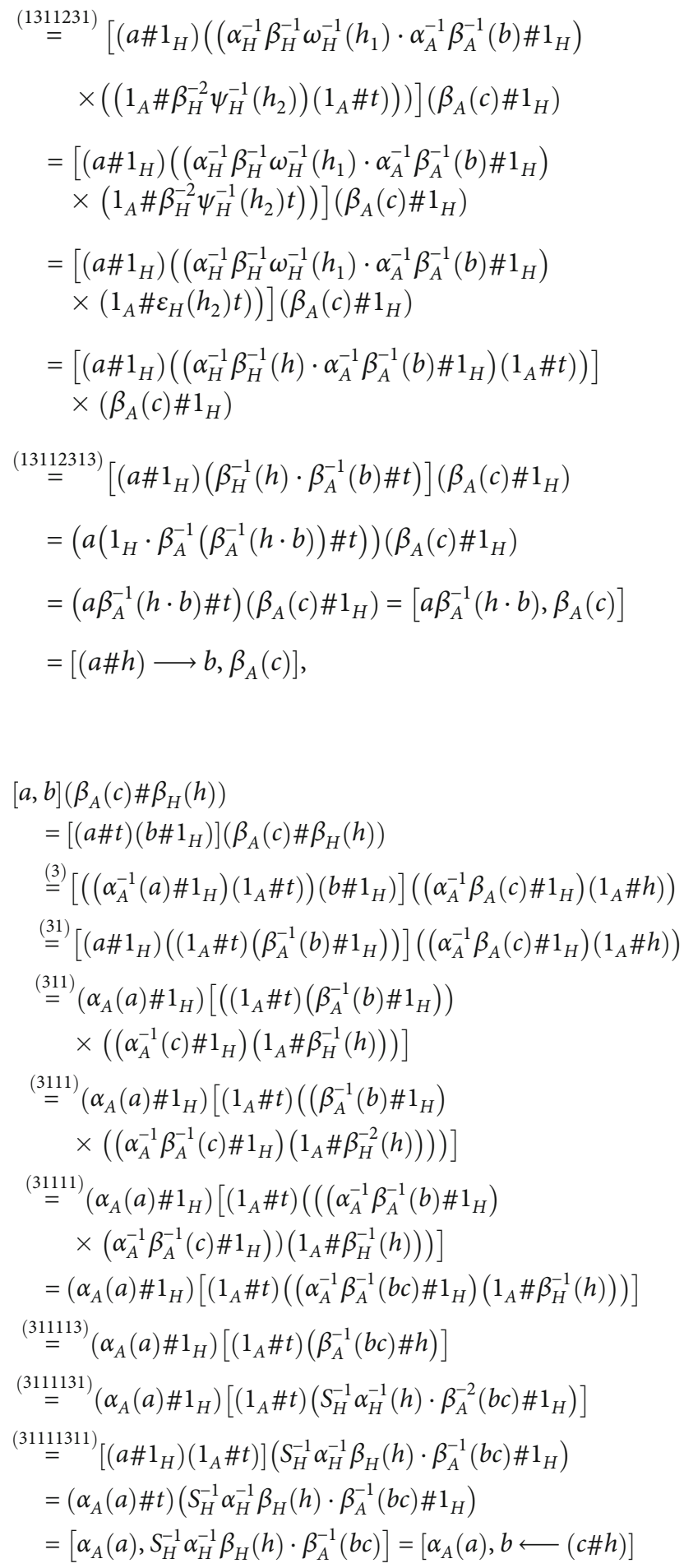

$$
\begin{aligned}
& =\left(a\left(\beta_{H}^{-1} \omega_{H}^{-1}\left(t_{1}\right) \cdot \beta_{A}^{-1}\left(a^{\prime}\right)\right) \# \psi_{H}^{-1}\left(t_{2}\right) 1_{H}\right)\left(\beta_{A}(b) \# 1_{H}\right) \\
& =\left(a \varepsilon_{H}\left(t_{1}\right) a^{\prime} \# \psi_{H}^{-1}\left(t_{2}\right) 1_{H}\right)\left(\beta_{A}(b) \# 1_{H}\right) \\
& =\left(a a^{\prime} \# t 1_{H}\right)\left(\beta_{A}(b) \# 1_{H}\right) \\
& =\left(a a^{\prime} \# t\right)\left(\beta_{A}(b) \# 1_{H}\right)=\left[a a^{\prime}, \beta_{A}(b)\right] .
\end{aligned}
$$

Next, since $A^{H}=t \cdot A$, thus the map $(-,-)$ is well defined. We now prove that $(-,-)$ is both an $\left(A^{H}, \alpha_{A}\right.$ ||$\left._{A^{H}},\left.\beta_{A}\right|_{A^{H}}\right)$-module map and middle $\left(A \# H, \alpha_{A} \otimes \alpha_{H}, \beta_{A} \otimes\right.$ $\beta_{H}$ )-linear. For any $a, b \in A, a^{\prime} \in A^{H}, h \in H$, we compute

$$
\begin{aligned}
\left(a^{\prime} a, \beta_{A}(b)\right)= & t \cdot \beta_{A}^{-1}\left(\left(a^{\prime} a\right) \beta_{A}(b)\right) \\
& \stackrel{(1)}{=} t \cdot \beta_{A}^{-1}\left(\alpha_{A}\left(a^{\prime}\right)(a b)\right) \\
= & t \cdot\left(\alpha_{A} \beta_{A}^{-1}\left(a^{\prime}\right) \beta_{A}^{-1}(a b)\right) \\
& \stackrel{(110)}{=}\left[\alpha_{H}^{-1} \omega_{H}^{-1}\left(t_{1}\right) \cdot \alpha_{A} \beta_{A}^{-1}\left(a^{\prime}\right)\right] \\
& \cdot\left[\beta_{H}^{-1} \psi_{H}^{-1}\left(t_{2}\right) \cdot \beta_{A}^{-1}(a b)\right] \\
= & \varepsilon_{H}\left(t_{1}\right) \alpha_{A}\left(a^{\prime}\right)\left(\beta_{H}^{-1} \psi_{H}^{-1}\left(t_{2}\right) \cdot \beta_{A}^{-1}(a b)\right) \\
= & \alpha_{A}\left(a^{\prime}\right)\left(\beta_{H}^{-1}(t) \cdot \beta_{A}^{-1}(a b)\right) \\
= & \alpha_{A}\left(a^{\prime}\right)\left(t \cdot \beta_{A}^{-1}(a b)\right)=\alpha_{A}\left(a^{\prime}\right)(a, b), \\
\left(\alpha_{A}(a), b a^{\prime}\right)= & t \cdot \beta_{A}^{-1}\left(\alpha_{A}(a)\left(b a^{\prime}\right)\right) \\
\stackrel{(1)}{=} & t \cdot \beta_{A}^{-1}\left((a b) \beta_{A}\left(a^{\prime}\right)\right) \\
= & t \cdot\left(\beta_{A}^{-1}(a b) a^{\prime}\right) \\
\stackrel{(110)}{=} & {\left[\alpha_{H}^{-1} \omega_{H}^{-1}\left(t_{1}\right) \cdot \beta_{A}^{-1}(a b)\right]\left[\beta_{H}^{-1} \psi_{H}^{-1}\left(t_{2}\right) \cdot a^{\prime}\right] } \\
= & {\left[\alpha_{H}^{-1} \omega_{H}^{-1}\left(t_{1}\right) \cdot \beta_{A}^{-1}(a b)\right] \varepsilon_{H}\left(t_{2}\right) \beta_{A}\left(a^{\prime}\right) } \\
= & \left(\alpha_{H}^{-1}(t) \cdot \beta_{A}^{-1}(a b)\right) \beta_{A}\left(a^{\prime}\right) \\
= & \left(t \cdot \beta_{A}^{-1}(a b)\right) \beta_{A}\left(a^{\prime}\right)=(a, b) \beta_{A}\left(a^{\prime}\right),
\end{aligned}
$$$$
\begin{aligned}
\left(a \longleftarrow(c \# h), \beta_{A}(b)\right)= & \left(S_{H}^{-1} \alpha_{H}^{-1} \beta_{H}(h) \cdot \beta_{A}^{-1}(a c), \beta_{A}(b)\right) \\
= & t \cdot \beta_{A}^{-1}\left[\left(S_{H}^{-1} \alpha_{H}^{-1} \beta_{H}(h) \cdot \beta_{A}^{-1}(a c)\right) \beta_{A}(b)\right] \\
= & t \cdot\left[\left(S_{H}^{-1} \alpha_{H}^{-1}(h) \cdot \beta_{A}^{-2}(a c)\right) b\right] \\
= & t \cdot\left[\left(S_{H}^{-1} \alpha_{H}^{-1} \psi_{H}^{-1}\left(h_{2}\right) \cdot \beta_{A}^{-2}(a c)\right)\left(\varepsilon_{H}\left(h_{1}\right) b\right)\right] \\
\stackrel{(3)}{=} & t \cdot\left[\left(S_{H}^{-1} \alpha_{H}^{-1} \psi_{H}^{-1}\left(h_{2}\right) \cdot \beta_{A}^{-2}(a c)\right)\right. \\
& \cdot\left(\left(S_{H}^{-1} \alpha_{H}^{-1} \beta_{H}^{-1} \omega_{H}^{-1} \psi_{H}^{-1}\left(h_{12}\right) \beta_{H}^{-2} \omega_{H}^{-2}\left(h_{11}\right)\right)\right. \\
& \left.\left.\cdot \beta_{A}^{-1}(b)\right)\right]
\end{aligned}
$$

$$
\begin{aligned}
& {\left[\alpha_{A}(a), a^{\prime} b\right]} \\
& \quad=\left(\alpha_{A}(a) \# t\right)\left(a^{\prime} b \# 1_{H}\right) \\
& \quad=\left(\alpha_{A}(a) \# t\right)\left(\left(a^{\prime} \# 1_{H}\right)\left(b \# 1_{H}\right)\right) \\
& \quad \stackrel{(1)}{=}\left((a \# t)\left(a^{\prime} \# 1_{H}\right)\right)\left(\beta_{A}(b) \# 1_{H}\right)
\end{aligned}
$$




$$
\begin{aligned}
& \stackrel{(32)}{=} t \cdot\left[\left(S_{H}^{-1} \alpha_{H}^{-1} \psi_{H}^{-2}\left(h_{22}\right) \cdot \beta_{A}^{-2}(a c)\right)\right. \\
& \cdot\left(\left(S_{H}^{-1} \alpha_{H}^{-1} \beta_{H}^{-1} \omega_{H}^{-1} \psi_{H}^{-1}\left(h_{21}\right) \beta_{H}^{-2} \omega_{H}^{-1}\left(h_{1}\right)\right)\right. \\
& \text { - } \left.\left.\beta_{A}^{-1}(b)\right)\right] \\
& \stackrel{(327)}{=} t \cdot\left[\left(S_{H}^{-1} \alpha_{H}^{-1} \psi_{H}^{-2}\left(h_{22}\right) \cdot \beta_{A}^{-2}(a c)\right)\right. \\
& \cdot\left(S_{H}^{-1} \beta_{H}^{-1} \omega_{H}^{-1} \psi_{H}^{-1}\left(h_{21}\right)\right. \\
& \text { - } \left.\left.\left(\beta_{H}^{-2} \omega_{H}^{-1}\left(h_{1}\right) \cdot \beta_{A}^{-2}(b)\right)\right)\right] \\
& \stackrel{(3276)}{=} t \cdot\left[\left(\alpha_{H}^{-1} \omega_{H}^{-1}\left(S_{H}^{-1}\left(\psi_{H}^{-1}\left(h_{2}\right)\right)_{1}\right)\right.\right. \\
& \text { - } \left.\beta_{A}^{-2}(a c)\right)\left(\beta_{H}^{-1} \psi_{H}^{-1}\left(S_{H}^{-1}\left(\psi_{H}^{-1}\left(h_{2}\right)\right)_{2}\right)\right. \\
& \text { - } \left.\left.\left(\beta_{H}^{-2} \omega_{H}^{-1}\left(h_{1}\right) \cdot \beta_{A}^{-2}(b)\right)\right)\right] \\
& \stackrel{(327610)}{=} t \cdot\left[S_{H}^{-1} \psi_{H}^{-1}\left(h_{2}\right)\right. \\
& \left.\cdot\left(\beta_{A}^{-2}(a c)\left(\beta_{H}^{-2} \omega_{H}^{-1}\left(h_{1}\right) \cdot \beta_{A}^{-2}(b)\right)\right)\right] \\
& \stackrel{(3276107)}{=}\left[t S_{H}^{-1} \psi_{H}^{-1}\left(h_{2}\right)\right] \\
& \text { · }\left(\beta_{A}^{-1}(a c)\left(\beta_{H}^{-1} \omega_{H}^{-1}\left(h_{1}\right) \cdot \beta_{A}^{-1}(b)\right)\right) \\
& =t \cdot\left(\beta_{A}^{-1}(a c)\left(\beta_{H}^{-1} \omega_{H}^{-1}\left(h_{1}\right) \varepsilon_{H}\left(h_{2}\right) \cdot \beta_{A}^{-1}(b)\right)\right) \\
& =t \cdot\left(\beta_{A}^{-1}(a c)\left(\beta_{H}^{-1}(h) \cdot \beta_{A}^{-1}(b)\right)\right) \\
& =t \cdot \beta_{A}^{-1}((a c)(h \cdot b)) \\
& \stackrel{(32761077)}{=} t \cdot \beta_{A}^{-1}\left(\alpha_{A}(a)\left(c \beta_{A}^{-1}(h \cdot b)\right)\right) \\
& =\left(\alpha_{A}(a), c \beta_{A}^{-1}(h \cdot b)\right) \\
& =\left(\alpha_{A}(a),(c \# h) \longrightarrow b\right) \text {. }
\end{aligned}
$$

Finally, the BiHom-associativity is obtained by

$$
\begin{aligned}
{[a, b] \longrightarrow \beta_{A}(c) } & =\left[(a \# t)\left(b \# 1_{H}\right)\right] \longrightarrow \beta_{A}(c) \\
& \stackrel{(7)}{=}\left(\alpha_{A}(a) \# t\right) \longrightarrow\left[\left(b \# 1_{H}\right) \longrightarrow c\right] \\
& =\left(\alpha_{A}(a) \# t\right) \longrightarrow\left(b \beta_{A}^{-1}\left(1_{H} \cdot c\right)\right) \\
& =\left(\alpha_{A}(a) \# t\right) \longrightarrow(b c)=\alpha_{A}(a) \beta_{A}^{-1}(t \cdot(b c)) \\
& =\alpha_{A}(a)\left(t \cdot \beta_{A}^{-1}(b c)\right)=\alpha_{A}(a)(b, c) \\
\alpha_{A}(a) \longleftarrow[b, c] & =\alpha_{A}(a) \longleftarrow\left[(b \# t)\left(c \# 1_{H}\right)\right] \\
& \stackrel{(7)}{=}(a \longleftarrow(b \# t)) \longleftarrow\left(\beta_{A}(c) \# 1_{H}\right) \\
& =\left[S_{H}^{-1} \alpha_{H}^{-1} \beta_{H}(t) \cdot \beta_{A}^{-1}(a b)\right] \longleftarrow\left(\beta_{A}(c) \# 1_{H}\right) \\
& =\left(t \cdot \beta_{A}^{-1}(a b)\right) \longleftarrow\left(\beta_{A}(c) \# 1_{H}\right) \\
& =1_{H} \cdot \beta_{A}^{-1}\left(\left(t \cdot \beta_{A}^{-1}(a b)\right) \beta_{A}(c)\right) \\
& =\left(t \cdot \beta_{A}^{-1}(a b)\right) \beta_{A}(c)=(a, b) \beta_{A}(c) .
\end{aligned}
$$

We get $\left[A^{H},{ }_{A^{H}} A_{A \# H, A \# H} A_{A^{H}}, A \# H\right]$ forms an associated Morita context.

\section{Data Availability}

There is no data available.

\section{Conflicts of Interest}

The authors declare that they have no conflicts of interest.

\section{Acknowledgments}

The authors sincerely thank the referee for his or her valuable suggestions and comments on this paper. This work was supported by the NSF of China (Nos. 11801515 and 12071441), the Natural Science Foundation of Zhejiang Province (No. LY20A010003), the Foundation of Zhejiang Educational Committee (No. Y201942625), and the Project of Zhejiang College, Shanghai University of Finance and Economics (No. 2020YJYB01).

\section{References}

[1] N. Aizawa and H. Sato, " $q$-Deformation of the Virasoro algebra with central extension," Physics Letters B, vol. 256, no. 2, pp. 185-190, 1991.

[2] N. Hu, " $q$-Witt algebras, $q$-Lie algebras, $q$-holomorph structure and representations," Algebra Colloquium, vol. 6, pp. 51-70, 1999.

[3] J. T. Hartwig, D. Larsson, and S. D. Silvestrov, "Deformations of Lie algebras using $\sigma$-derivations," Journal of Algebra, vol. 295, no. 2, pp. 314-361, 2006.

[4] D. Larsson and S. D. Silvestrov, "Quasi-hom-Lie algebras, central extensions and 2-cocycle-like identities," Journal of Algebra, vol. 288, no. 2, pp. 321-344, 2005.

[5] A. Makhlouf and S. D. Silvestrov, "Hom-algebra structures," Journal of Generalized Lie Theory and Applications, vol. 2, no. 2, pp. 51-64, 2008.

[6] S. Caenepeel and I. Goyvaerts, "Monoidal Hom-Hopf algebras," Communications in Algebra, vol. 39, no. 6, pp. 22162240, 2011.

[7] G. Graziani, A. Makhlouf, C. Menini, and F. Panaite, "BiHomassociative algebras, BiHom-Lie algebras and BiHom-bialgebras," Symmetry, Integrability and Geometry: Methods and Applications, vol. 11, article 086, p. 34, 2015.

[8] S. Guo, X. Zhang, and S. Wang, "Representations and deformations of Hom-Lie-Yamaguti superalgebras," Advances in Mathematical Physics, vol. 2020, Article ID 9876738, 12 pages, 2020.

[9] L. Liu, A. Makhlouf, C. Menini, and F. Panaite, " $\{\sigma, \tau\}$ -Rota-Baxter operators, infinitesimal Hom-bialgebras and the associative (Bi)Hom-Yang-Baxter equation," Canadian Mathematical Bulletin, vol. 62, no. 2, pp. 355-372, 2019.

[10] L. Liu, A. Makhlouf, C. Menini, and F. Panaite, "Rota-Baxter operators on $\mathrm{BiHom}$-associative algebras and related structures," Colloquium Mathematicum, vol. 161, no. 2, pp. 263294, 2020.

[11] L. Liu, A. Makhlouf, C. Menini, and F. Panaite, "BiHomNovikov algebras and infinitesimal BiHom-bialgebras," Journal of Algebra, vol. 560, pp. 1146-1172, 2020. 
[12] S. Wang, X. Zhang, and S. Guo, "Derivations and deformations of $\delta$-Jordan Lie supertriple systems," Advances in Mathematical Physics, vol. 2019, Article ID 3295462, 15 pages, 2019.

[13] R. K. Molnar, "Semi-direct products of Hopf algebras," Journal of Algebra, vol. 47, no. 1, pp. 29-51, 1977.

[14] S. Montgomery, Hopf Algebras and Their Actions on Rings, CBMS Lectures in Math, vol. 82, AMS, Providence, RI, 1993.

[15] N. Andruskiewitsch and H. J. Schneider, "On the classification of finite-dimensional pointed Hopf algebras," Annals of Mathematics, vol. 171, no. 1, pp. 375-417, 2010.

[16] Y. Chen, Z. Wang, and L. Zhang, "Integrals for monoidal Hom-Hopf algebras and their applications," Journal of Mathematical Physics, vol. 54, no. 7, article 073515, 2013.

[17] D. Yau, "Hom-bialgebras and comodule algebras," The International Electronic Journal of Algebra, vol. 8, pp. 45-64, 2010. 